\title{
Long term cognitive outcome in herpes simplex encephalitis
}

\author{
Firosh Khan ${ }^{1}$, Fazal Ghafoor ${ }^{2, *}$, Abdulkhader Shehna ${ }^{3}$ \\ ${ }^{1-3}$ Associate Professor, ${ }^{1,2}$ Dept. of Neurology, ${ }^{2}$ Dept. of Radiotherapy \& Oncology, ${ }^{1,2}$ MES Medical College, Perinthalmanna, \\ Malappuram, Kerala, 3Government Medical College, Thrissur, Kerala, India
}

*Corresponding Author:

Email: drfazalmes@yahoo.in

\begin{abstract}
Aims: Herpes Simplex Encephalitis (HSE) is a leading cause of acquired cognitive disability. We aimed to describe cognitive sequelae of HSE in relation to clinical variables during hospitalization.

Methods: Consecutive cases of encephalitis with a discharge diagnosis of HSE from January 2010 to December2016 were reviewed to collect the clinical details. Glasgow-Outcome-Score (GOS), Barthel-Index (BI), Seizure-Score (Engel-Score, ES) at the time of discharge, first follow-up and final follow-up were noted. A detailed neuropsychological evaluation was done at last follow-up in survivors and these scores were compared to clinical variables during hospitalization. $\chi^{2}$ test, Fisher's exact test and ANOVA were used for analysis.

Results: There were 30 patients (11 males) at a median age of 31.5 years. Cerebro-spinal-fluid was abnormal in $82.8 \%$, electroencephalogram in $96.7 \%$ and Magnetic Resonance Imaging (MRI) in 80\% patients. All were treated with acyclovir and $80 \%$ had intensive-care-unit stay. Mortality was $26.7 \%$ and it correlated with glasgow coma score <8, status epilepticus and Periodic Lateralized Epileptiform Discharges (PLEDs) in electroencephalogram. At the first follow-up of 8.4 \pm 5.2 weeks and final follow-up of $36.6 \pm 26.5$ months, survivors had a good functional recovery with GOS4-5 and BI90-100. At final follow-up, median ES was one. ES was not correlated with seizures, status-epilepticus, electroencephalogram or MRI features. At final follow-up, 5/18(27.8\%) had significant neuropsychological verified cognitive abnormalities and those with longest follow-up had least abnormalities. Neuropsychological profile didn't correlate with any clinical variables other than the association of poor attention score and orbito-frontal signal changes in MRI.

Conclusions: Acyclovir treated HSE has a mortality of $26.7 \%$, but survivors have good functional/seizure outcome. Significant cognitive problems occur in a third, but not related to any clinical variables.
\end{abstract}

Keywords: Encephalitis, Herpes, Outcome, Cognitive outcome, Neuropsychology.

\section{Introduction}

Viral encephalitis is probably the most debilitating infection of the brain parenchyma. ${ }^{1}$ The clinical syndrome depends on the affected virus and it is by virtue of the fact that each virus has a predilection for a particular area of the brain. ${ }^{2}$ Herpes Simplex Encephalitis (HSE) caused by the Herpes Simplex Virus (HSV), affecting mainly the temporal and orbitofrontal regions is one of the commonest sporadic viral encephalitis. ${ }^{3}$ Though HSV is treatable with the advent of antiviral agents, cognitive sequelae is not uncommon $^{4}$ and in fact may be becoming more apparent owing to increased survival. A study of the outcome, especially the cognitive aspect of HSE is really important since the management of cognitively impaired needs expertise and resources. ${ }^{5} \mathrm{We}$ aimed to evaluate the cognitive outcome of HSE treated in our center.

\section{Methods}

The objective was to review the cases of HSE treated in our center. One of the practical problems in HSE diagnosis was the confirmatory test. CerebroSpinal-Fluid (CSF) Polymerase Chain Reaction (PCR) for HSV is very specific, but the sensitivity is low. Like wise CSF antibody against HSV, Magnetic Resonance Image (MRI) features and electroencephalography
(EEG) findings are also less sensitive. So we resorted to a clinical method.

We reviewed all consecutive cases of encephalitis treated in our hospital from January 2010 to December 2016. Out of these, those with a final (discharge) diagnosis of HSE were selected for the study. Clinical diagnosis of Encephalitis was entertained if there was fever and altered sensorium with or without seizures and/or focal deficits. Those with positive CSF-PCR for HSV and/or positive CSF-HSV antibody titers were taken as definite cases of HSE, while those with characteristic temporal lobe MRI signal changes and/or characteristic periodic lateralized epileptiform discharges (PLEDs) in EEG were taken as probable cases of HSE.

A systematic chart review of selected cases was done to collect the demographic data, clinical features, investigations (blood and CSF analysis, brain computerized tomographic scan (CT), brain MRI, EEG etc.), treatment details and complications during hospital stay. The clinical and investigation details at first and final follow-up were also collected. A detailed neuropsychological testing was done at last follow up. The neuropsychological battery included tests for intellectual functions, memory functions, language functions, visuo-practic abilities and attention/executive functions (Table 1). Local versions of the tests (Malayalam language) were used. ${ }^{6}$ 


\section{Results}

Out of the 380 patients with a discharge diagnosis of encephalitis, $32(8.4 \%)$ met the inclusion criteria for HSE, but two were later excluded due to significant comorbidities affecting evaluation of cognitive function. Five met the criteria for confirmed HSE, whereas the rest were in the probable HSE category. The study group included 11 males and 19 females with a median age of 31.5 (range 9-64) years. Major presenting neurological symptoms included seizures, headache, altered sensorium and behavioral abnormalities. (Table 2)

Serum anti-HSV antibody was elevated in seven out of the eight patients (87.5\%) tested for the same. Twenty-nine patients had a CSF sample analyzed at a median of 10 (1-22) days of the onset of symptoms and at median zero $(-3-+10)$ days of starting acyclovir therapy; $24(82.8 \%)$ were abnormal: pleocytosis in $15(51.7 \%)$, presence of red blood cells in $21(72.4 \%)$, raised protein in $10(34.5 \%)$ and low sugar in $1(3.4 \%)$. CSF opening pressure was elevated in $5 / 14(35.7 \%)$. CSF anti-HSV antibody was positive in $1 / 15(6.7 \%)$, whereas the ratio of CSF/Serum anti-HSV antibody level was positive in $3 / 8(37.5 \%)$. CSF-HSV PCR was positive only in $2 / 9(22.2 \%)$ patients. Any of the above last three tests were done in 21 patients and six (28.6\%) were positive. No patient had all the three tests or any two tests simultaneously positive.

EEG was done at a median of 10.5 (1-22) days from onset of illness and $1(-3$ to +13$)$ day after starting acyclovir therapy; it was abnormal in 29 (96.7\%) patients. Thirty-two repeat EEGs were done on 19 patients and the major findings when initial and subsequent EEGs are taken together, are detailed in Table 3.

CT scan of the brain was done in 20 patients and was abnormal in $8(40 \%)$, four each with diffuse swelling and focal hypo-density. Brain MRI scans were done in 20 and was abnormal in 16 (80\%), with all showing focal hyper-intensity (HI) and one showing additional diffuse swelling. The focal $\mathrm{T}_{2}$ Weighted $\mathrm{HI}$ involved orbitofrontal $(n=3)$, medial-frontal $(n=4)$, frontal-convexity $(n=4)$, medial-temporal $(n=13)$, lateral-temporal $(n=8)$, and parietal $(n=7)$ regions. Bilateral changes were seen in $10(50 \%)$ patients, whereas CT showed bilateral changes only in one $(5 \%)$ patient.

Twenty-four patients (80\%) had intensive care unit (ICU) stay for a median of 5.5(3-149) days. Seizures occurred in 26/30 (86.7\%) during the acute stage, 14 of them having status epilepticus (SE): nine CSE, two NCSE and three with both CSE and NCSE. Out of these, seven patients had refractory status epilepticus (RSE) requiring 12-72 hours for control. Midazolam $(\mathrm{n}=8)$, propafol $(\mathrm{n}=2)$ and thiopentone $(\mathrm{n}=2)$ were used for the control. Other complications included dyselectrolytemia $(n=9)$, hyperglycemia $(n=5)$, renal dysfunction $(\mathrm{n}=4)$, hepatic dysfunction $(\mathrm{n}=9)$, cardiac dysrhythmia $(\mathrm{n}=2)$, myocardial ischemia $(\mathrm{n}=1)$, pneumonia $(n=9)$, adult respiratory distress syndrome $(n=4)$, urinary tract infection $(n=3)$ and deep vein thrombosis $(\mathrm{n}=2)$. Eleven patients $(36.7 \%)$ were mechanically ventilated for a median of 5.5 days and the indications included SE $(n=6)$, respiratory failure $(n=5)$, raised intracranial pressure $(n=4)$ and altered sensorium $(n=1)$. Tracheostomy was required in three patients and eight required prolonged (2-20 days) inotropic support.

All patients were initiated on acyclovir at a median of 7 (1-24) days from the symptom onset and was continued for a period of 10 days in eight, 14 days in fifteen and 21 days in one patient. The remaining six patients received it for a variable period of 1-13 days (till the time of their death). Ten had progressive worsening despite treatment and eight of them died; remaining 20 patients started improving after a median delay of 3 (2-12) days of initiation of acyclovir. Apart from acyclovir all patients received antiepileptic drugs (AEDs). Phenytoin $(n=28)$, carbamazepine $(n=12)$, phenobarbitone $(\mathrm{n}=11)$, clonazepam $(\mathrm{n}=8)$, clobazam $(\mathrm{n}=2)$, diazepam $(\mathrm{n}=1)$ and sodium-valproate $(\mathrm{n}=1)$ were the AEDs used. One patient received dexamethasone for initial three days as a part of the treatment for raised intracranial pressure.

There were eight $(26.7 \%)$ in hospital deaths. (Fig. 1) Two out of six (33.3\%) with sub-acute disease onset died as compared to six out of $24(25 \%)$ with acute onset of the symptoms. Hospital death was significantly associated with Glasgow Coma Score $(\mathrm{GCS})^{10}<8$ at presentation $\left(p=0.012, \chi^{2}\right.$ test). Six out of nine patients $(66.7 \%)$ with a GCS of $<8$ died during the hospital stay. Seven out of eight patients who died had either PLEDs or BiPLEDs in EEG while six had RSE suggesting that these two variables are also associated with hospital death. All eight patients who died had progressive worsening despite treatment; seven developed pneumonia and four had additional multi-organ failure.

First follow-up data was available in 22 and final follow-up data in 18 patients. The first follow-up was at a mean of $8.4 \pm 5.2$ weeks while the last follow-up was at a mean of $36.6 \pm 26.5$ months. All with follow-up data available had a good functional status at first (Glasgow outcome score; GOS 4-5) and final (GOS 5) follow-up. ${ }^{7}$ All except one had Barthel Index (BI) ${ }^{8}$ of 100 at final follow-up; the remaining one had a BI of 90. Major disabilities at final follow-up included seizures $(n=3)$, subtle intellectual problems $(n=2)$, dysarthria $(n=1)$, dysphasia $(n=3)$, subjective memory problems $(n=12)$, change in personality $(n=2)$ and irritability $(n=5)$. The median seizure score (Engel Score, ES) ${ }^{9}$ at final followup was one (range 0-7). Seven patients were seizure free and were off AEDs; eight were seizure free but on AEDs and the remaining three were having seizures despite medications. Ten patients had an EEG done at a median of 2.5 (2-31) months after discharge; three of 
these had focal temporal /fronto-temporal epileptiform discharges and two had focal temporal slowing.

The neuropsychological profile was examined at last follow-up in 18 patients. The domains tested were intellectual, memory, language, visuo-practic, attention and executive functions (Table 1). Unequivocal abnormalities were seen in five patients (Table 5). The cognitive performance of the three confirmed cases with follow-up didn't differ much from that of the probable cases.

Seizure score (Engel Score, ES) at last follow-up was not correlated with any clinical/electrographic seizures or status epilepticus (CSE or NCSE) during hospital stay. Similarly the score was not correlated with any PLEDs in EEG, BiPLEDs or any normal record (Table 6). The initial presentation with seizures or status epilepticus was also not related to final ES.

Abnormality in imaging was not correlated with hospital death or other outcome variables. Only one patient out of eight (12.5\%) with abnormal CT brain died during the hospital stay. The only patient with bilateral involvement in CT and nine out of ten with similar involvement in MRI survived. MRI scan of the brain was available in 18 patients and $14(77.8 \%)$ of them were abnormal. Four patients died, two with normal and two with abnormal MRI. On Fisher's exact test (two sided), the difference was not statistically significant $(p=0.197)$. Out of 18 patients with follow-up data available, MRI was done in 14 and twelve of them were abnormal. GOS was 5.0 in all 14 , while BI $(p=0.7$, ANOVA) or ES ( $p=0.584$, ANOVA) didn't differ significantly between those with normal and abnormal MRI. Seizure scores (ES) were significantly high in those patients with orbito-frontal (mean score 4.5, $p=0.003$, ANOVA) and occipital (mean score 4.0, $p=0.017$, ANOVA) signal changes in MRI. When neuropsychological scores at last follow-up were compared with MRI regional signal changes, orbitofrontal involvement was correlated with poor attention scores $(p=0.011$, ANOVA). None of the other neuropsychological scores were correlated with any MRI changes.

The mean delay in starting acyclovir treatment was 9.5 days from the illness onset; the delay was not significantly different in those who died in hospital (mean delay 7.71 days, $\mathrm{p}=0.641$, ANOVA). On Pearson correlation, the delay in starting acyclovir was not correlated with GOS, BI or ES. Similarly, the delay was not correlated with any of the neuropsychological 2 of them died, for 10 days in 8 , one of them died, for 21 days in one, who has survived and for a variable period in the rest six, but 4 of them died. The death rate was significantly more in those who received acyclovir for a nonstandard period $(p=0.047)$. When various durations of treatments were compared with neuropsychological scores of those who survived at last follow-up, there was no significant correlation. scores. Acyclovir was given for 14 days in 15 patients,

Out of nine patients with a GCS of $<8$ at presentation, three survived and they had no significant cognitive sequelae compared to others. Worsening at some point despite treatment was seen in 10 , seven of whom died, but the remaining had no significantly worse cognitive outcome. New focal deficits occurred in four and three of them died during hospital stay. Inotropic support was required in 8 , six of them died during hospital stay; the two patients survived had no significant sequelae. Eleven patients were mechanically ventilated and all the eight who subsequently died were in this group. Out of 14 patients with SE, seven had RSE, and six of them died. The person who survived had significant problem with visual and verbal memory as well as block design task. All four patients with anemia and three with hypernatremia died during the hospital stay. None of the parameters in CSF correlated with hospital mortality or cognitive outcome. Interestingly three patients who subsequently died had normal CSF study.

The disease onset was acute in 24 while sub-acute in the rest. The acuteness of disease onset had no correlation with hospital death or various neuropsychological scores in those who survived. The initial presentation was with headache in 10 , altered sensorium in six, seizures in 13 and behavioral problems in one. The pattern of initial presentation was not correlated to death in hospital $\left(\mathrm{p}=0.821, \chi^{2}\right.$ test) or to any of the neuropsychological scores in those who survived (ANOVA). Three presented with memory dysfunction and all of them had subjective memory problem on follow-up, apart from nine others. Two of them had abnormal digit symbols, picture completion and block design tasks while all three had logical memory and associative learning problems. Sixteen had behavioral changes at presentation, of which only three had persistent problems apart from two others without any behavioral problems at presentation.

Table 1: Neuropsychological Test Battery. WAIS Wechsler Adult Intelligence Scale; WMS - Wechsler Memory Scale

\begin{tabular}{|c|c|}
\hline Domain & Test battery \\
\hline $\begin{array}{l}\text { Intellectual } \\
\text { functions }\end{array}$ & $\begin{array}{l}\text { 1. WAIS information } \\
\text { 2. WAIS arithmetic } \\
\text { 3. WAIS digit symbols } \\
\text { 4. WAIS picture completion } \\
\text { 5. WAIS block design }\end{array}$ \\
\hline $\begin{array}{l}\text { Memory } \\
\text { functions }\end{array}$ & $\begin{array}{l}\text { 6. WMS logical memory } \\
\text { 7. WMS associative learning } \\
\text { 8. WMS visual reproduction } \\
\text { 9. Benton visual retention test } \\
\text { (BVRT) }\end{array}$ \\
\hline $\begin{array}{l}\text { Language } \\
\text { functions }\end{array}$ & $\begin{array}{l}\text { 10.Comprehension of } \\
\text { sentences with complex } \\
\text { semantic structures } \\
\text { 11.Confrontation naming of } \\
\text { pictures and body parts }\end{array}$ \\
\hline
\end{tabular}

IP Indian Journal of Neurosciences, January-March,2018;4(1):6-14 


\begin{tabular}{|l|l|}
\hline $\begin{array}{l}\text { Visuo-practic } \\
\text { abilities }\end{array}$ & $\begin{array}{l}\text { 12.Copy of a cube and Greek } \\
\text { cross } \\
\text { 13.Clock hand task }\end{array}$ \\
\hline $\begin{array}{l}\text { Attention and } \\
\text { executive } \\
\text { functions }\end{array}$ & $\begin{array}{l}\text { 14. WMS mental control } \\
\text { 15.The Stroop color naming } \\
\text { time } \\
\text { 16.The Stroop color-word } \\
\text { interference time } \\
\text { 17.Word fluency }\end{array}$ \\
\hline
\end{tabular}

Table 2: Major clinical features of Herpes Simplex Encephalitis

\begin{tabular}{|l|c|}
\hline Clinical features & $\begin{array}{c}\text { Number of } \\
\text { patients (\%) }\end{array}$ \\
\hline $\begin{array}{l}\text { Prodrome (myalgia, } \\
\text { coryza, diarrhoea) }\end{array}$ & $3(10.0)$ \\
\hline Fever & $26(86.7)$ \\
\hline Headache & $12(40.0)$ \\
\hline Altered sensorium & $17(56.7)$ \\
\hline Confusion & $8(26.7)$ \\
\hline Seizures & $26(86.7)$ \\
Partial & $17(56.7)$ \\
Generalized & $24(80.0)$ \\
Staus epilepticus & $5(16.7)$ \\
Epilepsia partialis & $1(3.3)$ \\
continua & $6(20.0)$ \\
\hline Hypotension & $3(10.0)$ \\
\hline Dysautonomia & $12(40.0)$ \\
\hline Bladder involvement & $10(33.3)$ \\
Incontinence & $2(6.7)$ \\
Retention & $2(6.7)$ \\
\hline Papilledema & $5(16.7)$ \\
\hline Release reflexes & \\
\hline
\end{tabular}

\begin{tabular}{|l|c|}
\hline Disinhibition & $2(6.7)$ \\
\hline Dysphasia & $9(30.0)$ \\
\hline Memory dysfunction & $3(10.0)$ \\
\hline Visual hallucinations & $3(10.0)$ \\
\hline Apathy & $7(23.3)$ \\
\hline Mutism & $8(26.7)$ \\
\hline Personality changes & $10(33.3)$ \\
\hline Hemiparesis & $4(13.3)$ \\
\hline Ataxia & $1(3.3)$ \\
\hline Abnormal movements & $2(6.7)$ \\
\hline Neck stiffness & $4(13.3)$ \\
\hline
\end{tabular}

Table 3: Major Electroencephalography (EEG) findings in Herpes Simplex Encephalitis

\begin{tabular}{|l|c|}
\hline EEG findings & $\begin{array}{c}\text { Number of } \\
\text { patients (\%) }\end{array}$ \\
\hline PLEDs & $11(36.7 \%)$ \\
\hline BiPLEDs & $9(30.0 \%)$ \\
\hline PLEDs or BiPLEDs & $18(60.0 \%)$ \\
\hline Focal / diffuse slowing & $10(33.3 \%)$ \\
\hline $\begin{array}{l}\text { Focal / generalized spike } \\
\text { waves }\end{array}$ & $8(26.7 \%)$ \\
\hline Nonspecific dysfunction & $5(16.7 \%)$ \\
\hline Electrographic seizures & $15(50.0 \%)$ \\
\hline $\begin{array}{l}\text { Convulsive status } \\
\text { epilepticus (CSE) }\end{array}$ & $2(6.7 \%)$ \\
\hline $\begin{array}{l}\text { Non-convulsive status } \\
\text { epilepticus (NCSE) }\end{array}$ & $5(16.7 \%)$ \\
\hline Any status epilepticus (SE) & $7(23.3 \%)$ \\
\hline Any normal record & $3(10.0 \%)$ \\
\hline
\end{tabular}

Table 4: Relationship of Electroencephalography (EEG) findings with seizure score (Engel Score) at final follow up ( $n=18$ patients)

\begin{tabular}{|l|c|c|c|}
\hline EEG variable & Number of patients & $\begin{array}{c}\text { Engel Score } \\
\text { (Mean/SD) }\end{array}$ & $\begin{array}{c}\text { P } \\
\text { (ANOVA) }\end{array}$ \\
\hline Any clinical or electrographic Seizures & 9 & $2.11 / 2.421$ & 0.083 \\
\hline Any status epilepticus & 3 & $3.0 / 2.0$ & 0.099 \\
\hline Any PLEDs & 6 & $0.67 / 0.516$ & 0.309 \\
\hline Any BiPLEDs & 4 & $1.75 / 2.217$ & 0.635 \\
\hline Any PLEDs / BiPLEDs & 9 & $1.11 / 1.537$ & 0.636 \\
\hline Any normal record & 3 & $0.67 / 0.577$ & 0.524 \\
\hline
\end{tabular}

Table 5: Neuropsychological profile of Herpes Simplex Encephalitis patients with abnormalities at last follow-up

\begin{tabular}{|c|c|}
\hline Patient details & Neuropsychological Profile \\
\hline $\begin{array}{l}\text { Patient no.2: 38-year-old female, febrile delirium. CSF pleocytosis. EEG } \\
\text { left temporal PLEDs / bi-temporal slowing, electrographic seizures. CT } \\
\text { normal MRI not done. Acyclovir started on } 4^{\text {th }} \text { day of illness and given for } \\
14 \text { days. No complications. Subjective dysphasia at last follow-up }\end{array}$ & $\begin{array}{l}\text { Poor language, intellectual, } \\
\text { memory, visuo-practic } \\
\text { functions; high color-word } \\
\text { errors }\end{array}$ \\
\hline $\begin{array}{l}\text { Patient no.13: 50-year-old male, at admission GCS 8, GTCS. CSF } \\
\text { pleocytosis, HSV antibody negative. EEG left temporal spike waves, } \\
\text { seizures. CT not done, MRI T2 WI hyper-intensity left mesial temporal. } \\
\text { Acyclovir started on 3rd day of illness, given for } 14 \text { days. Hypoglycemia } \\
\text { due to diabetes. Subjective memory problems at last follow-up }\end{array}$ & $\begin{array}{l}\text { Poor delayed visual } \\
\text { reproduction }\end{array}$ \\
\hline
\end{tabular}




\begin{tabular}{|c|c|}
\hline $\begin{array}{l}\text { Patient no.16: 46-year-old male, at admission GCS 12, GTCS. CSF sugar } \\
\text { low, HSV antibody negative. EEG right temporal electrographic seizures. } \\
\text { CT not done. MRI T2 WI hyper-intensity bilateral orbito-frontal, parietal, } \\
\text { occipital regions. Acyclovir initiated on } 24^{\text {th }} \text { day of illness, given for } 3 \text { days } \\
\text { only. At the last follow-up seizures, intellectual problems, dysphasia, } \\
\text { memory problems and irritable behavior }\end{array}$ & $\begin{array}{l}\text { Poor digit symbols, picture } \\
\text { completion, block design, } \\
\text { logical memory, paired } \\
\text { associative learning, visual } \\
\text { reproduction }\end{array}$ \\
\hline $\begin{array}{l}\text { Patient no.25: } 28 \text {-year-old male, at admission GCS } 8 \text {, GTCS. CSF normal. } \\
\text { EEG right temporal spike waves, BiPLEDs, slowing, clinical and } \\
\text { electrographic seizures. CT normal, MRI not done. Acyclovir started on } 7^{\text {th }} \\
\text { day of illness, given for } 14 \text { days. He had refractory status epilepticus, was in } \\
\text { ICU and was on mechanical ventilation. At last follow-up, mild subjective } \\
\text { memory problems }\end{array}$ & $\begin{array}{l}\text { Poor block design, delayed } \\
\text { logical memory, paired } \\
\text { associative learning, visual } \\
\text { reproduction }\end{array}$ \\
\hline $\begin{array}{l}\text { Patient no.27: 54-year-old male, febrile delirium, GCS 12, GTCS. CSF } \\
\text { normal, PCR and HSV antibody normal. EEG right fronto-temporal PLEDs, } \\
\text { electrographic seizures. CT not done, MRI right temporal (mesial and } \\
\text { lateral) T2 WI hyper-intensities. Acyclovir started on 5th day of illness, } \\
\text { given for } 21 \text { days. In ICU for } 2 \text { weeks. At last follow-up seizure free, } \\
\text { subjective memory and intellectual problems }\end{array}$ & $\begin{array}{l}\text { Poor block design, delayed } \\
\text { logical memory, visual } \\
\text { reproduction }\end{array}$ \\
\hline
\end{tabular}

Table 6: Residual Disabilities of Herpes Simplex Encephalitis at last follow-up

\begin{tabular}{|l|c|}
\hline \multicolumn{1}{|c|}{ Disability } & Number of patients (\%) \\
\hline Subjective memory dysfunction & $12(66.7)$ \\
\hline Behavioral problems (only irritability) & $5(27.8)$ \\
\hline Seizures (all partial) & $3(16.7)$ \\
\hline Dysphasia & $3(16.7)$ \\
\hline General intellectual problems & $2(11.1)$ \\
\hline Dysarthria & $1(5.6)$ \\
\hline
\end{tabular}
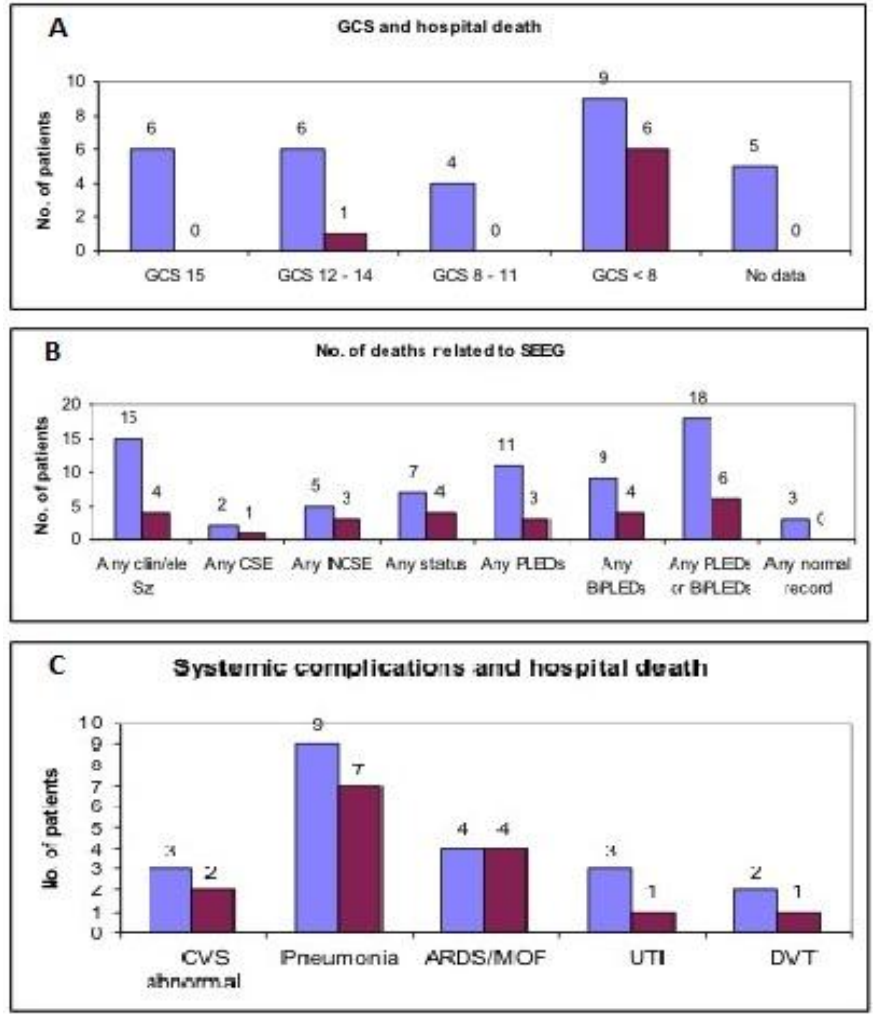

Fig. 1 

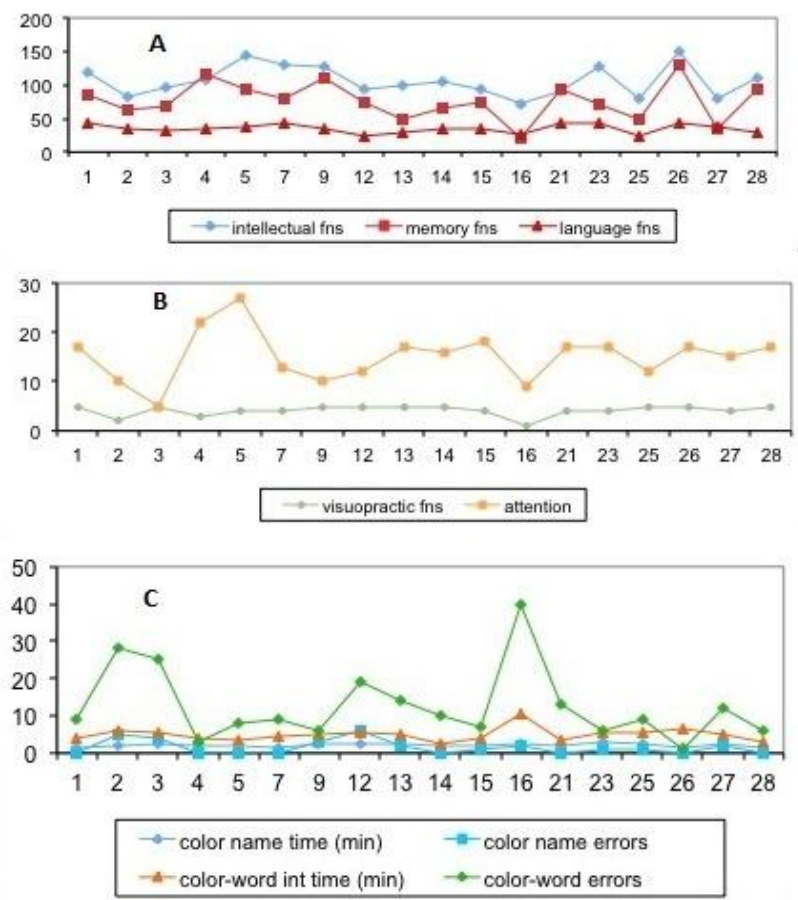

Picture.1:Grahical representation of neuropsychological test results

Fig. 2

\section{Discussion}

It is a common observation in our center that a sizeable number of sporadic encephalitis patients behave like herpes encephalitis clinically, electrographically, radiologically and more importantly in the response to acyclovir treatment, despite negative confirmatory tests. Many of our patients have financial constraints for HSV-PCR testing and if at all done, the result of the test is delayed so that the clinical decision is more important in starting treatment. So in our institution, HSE is clinically diagnosed and treatment is initiated. At the time of discharge, treating physician will code the final diagnosis based on the clinical diagnosis and the results of ancillary tests. We selected consecutive cases with final diagnosis of HSE and that's why we have wider inclusion criteria in our study.

The age of patients in our cohort ranged from 9 to 64 years). Bimodal age distribution noted previously ${ }^{11}$ was not observed in the current study. Though $80 \%$ of the patients had an acute onset, this was not correlated with either hospital death or the neuropsychological profile (in those who survived). The same was true with the correlation of the pattern of presenting neurological/systemic symptoms and hospital death/neuropsychological profile. The lower incidence of prodromal symptoms and the higher incidence of initial systemic symptoms as observed in the current study is comparable to previous studies. ${ }^{11}$ Similarly the overall incidence of personality changes (33.3\%), mutism (26.7\%), apathy (23.3\%), dysphasia (20\%), hemiparesis $(13.3 \%)$, memory dysfunction (10\%), visual hallucination (10\%) and ataxia (3.3\%) also match to what is described earlier. ${ }^{11}$ However, seizures $(86.7 \%)$ and altered sensorium $(83.3 \%)$ are significantly more in the current study. Our hospital is a quaternary referral center caring severe cases and that may be the reason for this.

In tune with previous studies, CSF examination was abnormal in a high proportion of patients. Though the proportion of cases with elevated opening pressure, elevated proteins, pleocytosis and low sugar were similar to reported literature, the incidence of RBCs in CSF was high $(72.4 \%)$ in our cohort. The exact reason for this is not sure. CSF to serum anti HSV antibody ratio was positive in only $37.5 \%$ of patients. This is not surprising since the reported sensitivity of this test is only around $50 \%$, though the specificity is more than $80 \%$. $^{12}$ The low sensitivity (22.2\%) of CSF-PCR in the current study is surprising since the reported sensitivity is as high as $95-98 \% .{ }^{13,14}$ The PCR remains positive for at least five days after starting acyclovir therapy. ${ }^{15,16}$ Hence the mean delay here of 2.4 days cannot explain the finding. Presence of RBCs in CSF may interfere with the test, ${ }^{17}$ however the two CSF samples, which were positive also had RBCs in it, one having more than $1000 / \mathrm{mm}^{3}$. So the exact cause of this low sensitivity of CSF-PCR in the current study remains unexplained. However negative PCR test is not rare and despite several explanations, the issue is unsettled. ${ }^{18}$ 
EEGs were recorded abnormal in $96.7 \%$ patients similar to the $95 \%$ abnormality reported in a previous study. ${ }^{19}$ When all EEGs are considered together, clinical/electrographic seizures were seen in 50\% patients, PLEDs or BiPLEDs in $60 \%$ and any status epilepticus $23.3 \%$. Three patients had at least one normal EEG and they all had favorable outcome with GOS 5 and BI 100 at last follow-up. One patient had recovering dysphasia, poor performance in picture completion, block design, copying and clock hand tasks while another one had an ES of one and subjective memory dysfunction at last follow-up but fared normally in the formal neuropsychological assessment. On statistical analysis the seizure score at last follow-up or any other outcome variables were not significantly correlated to any of the EEG parameter. This finding appears similar to the study by Lai and Gragasin who concluded that EEG does not predict the survival or severe disability. ${ }^{20}$ However, the study by Siren et al showed that PLEDs, delta bursts and focal discharges were correlated with poor outcome. ${ }^{19}$

Overall three patients had no CT or MRI studies, seven had only CT (two abnormal), seven had only MRI (six abnormal) and thirteen had both CT and MRI (ten abnormal). So 18 out of $27(66.7 \%)$ with any neuroimaging had an abnormality, in tune with $75 \%$ sensitivity reported in literature. ${ }^{21}$ The sensitivity of CT scan was only $40 \%$, in sharp contrast to the $70-100 \%$ sensitivity reported in other studies. ${ }^{22,23}$ MRI sensitivity was $80 \%$ and it picked $57.1 \%$ of CT negative cases showing that MRI is superior to CT scan in HSE and underscores the suggestion that MRI is the imaging modality of choice in HSE. ${ }^{1,}{ }^{24}$ The most common site involved was medial temporal region $(81.3 \%)$, similar to other series. ${ }^{(23)}$ Common extra-temporal sites included frontal and parietal regions. ${ }^{21}$

Hospital death was not correlated with any MRI abnormality. Out of 18 patients with follow-up data available, MRI was done in 14, and twelve of them were abnormal. GOS, BI or ES didn't differ significantly between those with normal and abnormal MRI. However seizure scores were significantly high in those patients with orbitofrontal and occipital signal changes in MRI. This finding should be interpreted with caution since only two had the abnormality. When neuropsychological scores at last follow-up were compared with MRI regional signal changes, orbitofrontal involvement was correlated with poor attention scores; again the small sample size may be a confounding factor. None of the other neuropsychological scores were correlated with any MRI changes.

Status epilepticus (SE) was a major complication and seven had refractory status epilepticus (RSE). Out of these seven, five died within one month. Though the other two survivors had GOS 5 at three months, both had seizures and neuropsychological dysfunction at last follow-up, suggesting that RSE is a poor prognostic factor in HSE. Similarly requirement of inotropic support and requirement of ventilator support for raised intracranial pressure were negative prognostic factors. The former occurred in eight patients, and out of them six $(75 \%)$ died. The latter occurred in four; all of them (100\%) developed new focal deficits and died by one month. Three patients had cardiac dysrhythmia/coronary ischemia and all of them subsequently died. All the seven patients who died in the hospital had pneumonia and four of them additionally had ARDS/MOF. All of them had metabolic disturbances as well. Twenty-four patients (80\%) had ICU stay for a mean of 15.4 (3-149) days, but the length of ICU stay was not correlated with hospital death. Eleven patients were given mandatory ventilation for a mean of 23.5 days; again the duration of mandatory ventilation was not correlated with hospital mortality.

Acyclovir was given to all patients and the mean delay in starting therapy was 9.5 days. The delay in initiating treatment was not correlated with hospital death, functional status, seizure score or any of the neuropsychological variables. Acyclovir was given for 7,14 or 21 days in most patients (standard regimen). Though the death rate was significantly more in those who received acyclovir for a nonstandard period, this is artifactual since those are the patients who mostly died before completing the regimen. The duration of acyclovir treatment didn't correlate with neuropsychological profile as well.

The GOS was 4-5 (mean 4.5) at first follow-up. Similarly at last follow-up, the outcome was good with GOS 5 in all and BI 100 in all except one who had a score of 90. The residual disabilities at last follow-up are given below (Table 6). However, none of these disabilities were correlated with EEG or clinical features. Multiple self/attendant reported behavioral abnormalities were noted in a previous study. ${ }^{25}$ But in the current study the report is less, possibly due to under reporting since standard scales were not used.

The neuropsychological tests used were to cover the intellectual, language, memory, visuo-practic, attention and executive functions (Table 1), the domains reported to be abnormal in previous studies. ${ }^{26}$ Screening mini-mental-status examination (MMSE) was not done since it is documented that MMSE will not reflect abnormalities in neuropsychological testing. ${ }^{5}$ Various test results are represented graphically below (Fig. 2). Maximum abnormalities were with patient no. $2,13,16,25$ and 27; main features of these patients are summarized in Table 5 .

Overall, it can be observed that the person (Patient no.16) who had delayed treatment initiation and who received acyclovir only for 3 days has maximum sequelae. However, both these factors were not significant as far as the whole study is concerned. The abnormalities are milder in those with longer follow-up. This may indirectly suggest progressive improvement 
in cognitive, behavioral and seizure outcomes in patients with HSE. This is in accordance with the study by Hokkanen et al, ${ }^{26}$ where the cognitive symptoms after encephalitis improved in $73 \%$ of the patients. They concluded that in most patients, the cognitive decline has taken place already at the acute stage and further decline was unusual. However, unlike in their study where two-third with persistent cognitive problems had frequent seizures, only one (Patient no.16) of our patients with cognitive problems had frequent seizures.

Intellectual functions: Scaled scores were used for comparison. Abnormally low values were seen with patient no. 2, 12, 16, 25 and 27. Similar abnormalities had been reported in the study by Hokkanen et al. ${ }^{26}$ In that study, significant abnormality was in digit symbols while in the current study, the abnormalities were more in the block design, information and arithmetic subtests. Language abilities: Two patients (no.12 and 25) were poor in comprehension of sentences with complex semantic structures. Patient no.12 had MRI evidence of involvement of bilateral medial frontal and left temporal (lateral more than medial, only patient in the study having such a picture) regions. Though the other patient had no MRI study, his EEG showed bi-temporal PLEDs. This may suggest that temporal especially lateral temporal involvement may be the cause for semantic dysfunction. All patients fared well in confrontation naming of pictures and body parts except one mistake made by patient no.16. This is in contrary to previous studies, which report a higher rate of abnormalities. Pietrini et al described naming problems (especially of living objects) in two patients using 30 pictures of plants, animals and objects. ${ }^{27}$ Kapur et al reported naming deficit in four out of ten patients. ${ }^{28}$ Barbarotto et al used 60 items (fruits, vegetables, animals, furniture, vehicles and tools) and found abnormality in four out of seven patients. ${ }^{29}$ Use of high frequency items and smaller number of items may be the cause for lack of sensitivity of these tests in the current study.

Memory functions: Verbal memory was involved in fourteen, but maximum affected in seven patients (no.3,12,13,15,16,25,27) and visual memory affected in six (no. $2,13,14,16,25,27)$. Overall memory impairment was observed in $14 / 18(77.8 \%)$ of the patients (other than no.1,9,21,26). This holds well with the results of the $70 \%$ involvement in the study by McGrath et al. ${ }^{25}$ In the study by Hokkanen et al visual memory was impaired in 59\% while verbal memory in $76 \% .^{30}$ This major involvement of verbal memory is duplicated in the current study as well. Normal semantic memory in the presence of impaired short term and episodic memory is well described in HSE. ${ }^{28}$ Though this is there in the current study as well, the lack of sensitivity of the test items used for semantic naming tasks should be kept in mind.
Visuopractic abilities: Maximum abnormalities were seen in patient no.2 and 16. Generally, the involvement will be less in HSE. A study by Hokkanen et al showed no significant changes. ${ }^{26}$ Bilateral parietal and occipital regions were involved in the patient no.16, which could account for the abnormalities; no MRI was done in the other patient. How far the abnormalities are contributed by attention problems is unknown. Other parietal functions like calculation and praxis will be normal in HSE as reported in two patients by Pietrini et al. ${ }^{27}$

Attention and executive functions: Attention deficit was maximum with patient no. $3,9,16,25$. During the stroop test, maximum time was taken and maximum errors were committed by patient no. 2,12,16,27. Similar result is reported by Hokkanen et al. ${ }^{26}$ Gordon et al has reported frontal lobe dysfunction in one out of four patients they studied. ${ }^{5}$ The abnormality in attention may contribute to performance in other tests.

Herpes simplex encephalitis has been reported as a cause for dementia. ${ }^{31}$ However, in the current study, according to the DSM IV criteria for dementia, only patient no.27 may satisfy the diagnosis, since he had significant impairment in the social and occupational functioning. However, like the other patients, on long term he is likely to improve and so whether he can be called demented is doubtful. Overall the cognitive outcome of patients in this study appear to be better compared to previous studies. ${ }^{5,}{ }^{28}$ In this respect, the current study is similar to the study by Hokkanen et $\mathrm{al}^{26}{ }^{26}$ where only two out of eight patients with HSE had persistent cognitive deficits.

The neuropsychological profile was not correlated with delay in initiating the acyclovir treatment. However, this should be taken with caution since the numbers were small. Also out of the five patients with significant neuropsychological abnormality, the patient who presented late had the worst scores. It has been argued that the acyclovir treatment decreases the mortality but increases the relative frequency of cognitive deficits in the survivors. ${ }^{32}$ It was not possible to test this in the current study since all the patients received acyclovir.

\section{Conclusions}

Herpes simplex encephalitis is a leading cause of neurological disability worldwide. In this study, though the mortality is around $30 \%$, the outcome is fair with significant cognitive problems occurring in only a third of those who survived. Even they are likely to recover on long follow-up. Epilepsy occurred only in a minority and those with orbitofrontal and occipital signal changes in MRI had more chance for the same. Orbitofrontal involvement was associated with poor attention scores as well. Refractory status epilepticus, requirement of inotropic support, requirement of ventilator support, cardiac dysrhythmia/coronary ischemia, pneumonia and ARDS/MOF during the acute stage are associated with mortality. 


\section{References}

1. Baringer JR. Herpes simplex infections of the nervous system. Neurol Clin.2008;26(3):657-74.

2. Sili U, Kaya A, Mert A; HSV Encephalitis Study Group. Herpes simplex virus encephalitis: clinical manifestations, diagnosis and out come in 106 adult patients. J Clin Virol. 2014;60(2):112-8.

3. Kohl S. Herpes Simplex Virus. In: Behrman RE, Kliegman RM, Jenson HB. Behrman: Nelson Textbook of Pediatrics. 17th ed. Philadelphia: Saunders;2004.

4. Utley TF, Ogden JA, Gibb A, McGrath N, Anderson NE. The long-term neuropsychological outcome of herpes simplex encephalitis in a series of unselected survivors. Neuropsychiatry Neuropsychol Behav Neurol. 1997 Jul. 10(3):180-9.

5. Gordon B, Selnes OA, Hart J Jr, Hanley DF, Whitley RJ. Long-term cognitive sequelae of acyclovir-treated herpes simplex encephalitis. Arch Neurol. 1990;47(6):646-7.

6. http://www.cogneurology-ian.in/malayalam accessed 1st December 2016.

7. Jennett B; Bond M. Assessment of outcome after severe brain damage. Lancet.1975;1(7905):480-484.

8. Mahoney F. Barthel D. Functional evaluation: the Barthel Index. Md Med J.1965;14:61-65.

9. Engel J, Van Ness PC, Rasmussen TB, Ojemann LM. Outcome with respect to epileptic seizures. In: Engel J, ed. Surgical Treatment of the Epilepsies. 2nd ed. New York, NY: Raven Press;1993:609-621.

10. Teasdale G, Jennett B. Assessment of coma and impaired consciousness. A practical scale. Lancet.1974;2(7872):81-4.

11. Marton R, Gotlieb-Steimatsky T, Klein C, Arlazoroff A. Acute herpes simplex encephalitis: clinical assessment and prognostic data. Acta Neurol Scand. 1996;93(23):149-55.

12. Bos CA, Olding-Stenkvist E, Wilterdink JB, Scheffer AJ. Detection of viral antigens in cerebrospinal fluid of patients with herpes simplex virus encephalitis. J Med Virol. 1987;21(2):169-78.

13. Cinque P, Bossolasco S, Lundkvist A. Molecular analysis of cerebrospinal fluid in viral diseases of the central nervous system. J Clin Virol. 2003;26(1):1-28.

14. Lakeman FD, Whitley RJ. Diagnosis of herpes simplex encephalitis: application of polymerase chain reaction to cerebrospinal fluid from brain-biopsied patients and correlation with disease. National Institute of Allergy and Infectious Diseases Collaborative Antiviral Study Group. J Infect Dis. 1995;171:857-863.

15. Aurelius E, Johansson B, Sköldenberg B, Staland A, Forsgren M. Rapid diagnosis of herpes simplex encephalitis by nested polymerase chain reaction assay of cerebrospinal fluid. Lancet. 1991;337(8735):189-92.

16. Cinque P, Cleator GM, Weber T, Monteyne P, Sindic CJ, van Loon AM. The role of laboratory investigation in the diagnosis and management of patients with suspected herpes simplex encephalitis: a consensus report. The EU Concerted Action on Virus Meningitis and Encephalitis. J Neurol Neurosurg Psychiatry. 1996;61(4):339-45.

17. Linde A, Klapper PE, Monteyne P, Echevarria JM, Cinque P, Rozenberg F, Vestergaard BF, Ciardi M, Lebon P, Cleator GM. Specific diagnostic methods for herpesvirus infections of the central nervous system: a consensus review by the European Union Concerted Action on Virus Meningitis and Encephalitis. Clin Diagn Virol. 1997;8(2):83-104.

18. Adler AC, Kadimi S, Apaloo C, Marcu C. Herpes simplex encephalitis with two false-negative cerebrospinal fluid PCR tests and review of negative PCR results in the clinical setting. Case Rep Neurol. 2011;3(2):172-8.

19. Sirén J, Seppäläinen AM, Launes J. Is EEG useful in assessing patients with acute encephalitis treated with acyclovir? Electroencephalogr Clin Neurophysiol.1998;107(4):296-301.

20. Lai CW, Gragasin ME. Electroencephalography in herpes simplex encephalitis. J Clin Neurophysiol. 1988;5(1):87103.

21. Wasay M, Mekan SF, Khelaeni B, Saeed Z, Hassan A, Cheema Z, et al. Extra temporal involvement in herpes simplex encephalitis. Eur J Neurol. 2005;12(6):475-9.

22. Hindmarsh T, Lindqvist M, Olding-Stenkvist E, Sköldenberg B, Forsgren M. Accuracy of computed tomography in the diagnosis of herpes simplex encephalitis. Acta Radiol Suppl. 1986;369:192-6.

23. Demaerel P, Wilms G, Robberecht W, Johannik K, Van Hecke P, Carton H, Baert AL. MRI of herpes simplex encephalitis. Neuroradiology. 1992;34(6):490-3.

24. Schroth G, Gawehn J, Thron A, Vallbracht A, Voigt K. Early diagnosis of herpes simplex encephalitis by MRI. Neurology. 1987;37(2):179-83.

25. McGrath N, Anderson NE, Croxson MC, Powell KF. Herpes simplex encephalitis treated with acyclovir: diagnosis and long term outcome. J Neurol Neurosurg Psychiatry. 1997;63(3):321-6.

26. Hokkanen L, Launes J. Cognitive recovery instead of decline after acute encephalitis: a prospective follow up study. J Neurol Neurosurg Psychiatry. 1997;63(2):222-7.

27. Pietrini V, Nertempi P, Vaglia A, Revello MG, Pinna V, Ferro-Milone F. Recovery from herpes simplex encephalitis: selective impairment of specific semantic categories with neuroradiological correlation. J Neurol Neurosurg Psychiatry. 1988;51(10):1284-93.

28. Kapur N, Barker S, Burrows EH, Ellison D, Brice J, Illis LS, Scholey K, Colbourn C, Wilson B, Loates M. Herpes simplex encephalitis: long term magnetic resonance imaging and neuropsychological profile. J Neurol Neurosurg Psychiatry. 1994;57(11):1334-42.

29. Barbarotto R, Capitani E, Laiacona M. Naming deficit in herpes simplex encephalitis. Acta Neurol Scand. 1996;93(4):272-80.

30. Hokkanen L, Launes J. Neuropsychological sequelae of acute-onset sporadic viral encephalitis. Neuropsychol Rehabil. 2007;17(4-5):450-77.

31. Steel AJ, Eslick GD. Herpes Viruses Increase the Risk of Alzheimer's Disease: A Meta-Analysis. J Alzheimers Dis. 2015;47(2):351-64.

32. Greenwood R, Bhalla A, Gordon A, Roberts J. Behaviour disturbances during recovery from herpes simplex encephalitis. J Neurol Neurosurg Psychiatry. 1983;46(9):809-17. 